\title{
Specific Health Guidance, the Nationwide Lifestyle Intervention Program Targeting Metabolic Syndrome, Seems to be Successful in Japan
}

\author{
Yuji Matsuzawa
}

Sumitomo Hospital, Osaka, Japan

\section{See article vol. 25: 308-322}

The concept of metabolic syndrome has been proposed by several committees, although there has been considerable disagreement over the definition and diagnostic criteria. The first definition and criteria referring to abdominal obesity was proposed by the National Cholesterol Education Program Adult Treatment (ATP III) in $2001^{1)}$. ATP III adopted abdominal obesity estimated by waist circumference (WC) rather than by body mass index (BMI) as one of five factors, in addition to hypertriglyceridemia, low HDL-cholesterol, high blood pressure, and hyperglycemia, as the basis of establishing metabolic syndrome, although ATP III did not require abdominal obesity as an essential component. In 2005, the International Diabetes Foundation (IDF) reported a new definition and diagnostic criteria of metabolic syndrome, making abdominal obesity an essential factor required in the diagnosis, with particular emphasis on waist measurement as a single screening tool ${ }^{2)}$. Since the definition and diagnostic criteria were proposed by the IDF, a general agreement seemed to have been reached that metabolic syndrome be designated as a multiple risk factor syndrome induced by abdominal obesity. However, controversies on the significance and cutoff point of WC have occurred after the IDF report. These controversies may have arisen from a misunderstanding of the purpose and significance of the measurement of WC, which is supposed to be a surrogate marker of visceral adiposity measured by CT scan. In these previous criteria, the significance of WC and the scientific basis of its cutoff point were not clearly indicated, and there were no

Address for correspondence: Yuji Matsuzawa, Sumitomo Hospital, Osaka, Japan

E-mail: matsuzawa-yuji@sumitomo-hp.or.jp

Received: January 5, 2018

Accepted for publication: January 5, 2018 data of visceral adiposity measured by CT scan as a golden standard, although they considered abdominal obesity as visceral obesity. The representatives of the IDF, the International Atherosclerosis Society, and the American Heart Association/National Heart, Lung and Blood Institute held discussions in an attempt to resolve the remaining differences in the definition of metabolic syndrome and agreed that abdominal obesity should not be a prerequisite for the diagnosis but that it is one of five risk factors, requiring the presence of any three of five factors. They also suggested that abdominal obesity should be defined based on the national cutoff point of each country.

However, in Japan, where accumulated data for visceral adiposity have been measured by CT scan, metabolic syndrome has been designated to be a multiple risk factor syndrome caused by visceral fat accumulation, and in such cases lifestyle modification to reduce visceral fat should take priority over drug treatment for each risk factor. The Japanese Committee for the definition and diagnostic criteria of metabolic syndrome adopted a cutoff point of $100 \mathrm{~cm}^{2}$ for visceral fat area for both men and women because the risk of metabolic syndrome increases over this point in both men and women. WC that corresponded to visceral fat of $100 \mathrm{~cm}^{2}$ was $85 \mathrm{~cm}$ in men and $90 \mathrm{~cm}$ in women ${ }^{3)}$. Although many different cutoff points have been adopted by different organizations and countries, the cutoff point in Japan is the only one that is determined using visceral fat area thresholds for morbidity.

According to the Japanese criteria, the Japanese Government started a new health policy by providing specific health checkups followed by specific counseling for subjects diagnosed with metabolic syndrome since 2008. The first official descriptive analysis of this nationwide lifestyle intervention program to improve the risk factors for metabolic syndrome in nonmedicated adults $40-74$ years of age was reported by Tushita $e t a l^{4)}$. They found that the improvements in obe- 
sity indices, including WC, BMI, and body weight, were significantly greater in participants of specific health guidance (SHG) than in nonparticipants. Sustainable BMI and WC reductions in SHG participants accompanied similar sustainable improvements in TG, HDL cholesterol, blood pressure, and initial reductions in HBA1c. Furthermore, the proportion of individuals who started pharmacological therapy in each follow-up year was significantly smaller in SHG participants than in nonparticipants. The results from these analyses demonstrate that lifestyle modification to reduce visceral fat is effective in the selected subjects with increased visceral adiposity, so-called metabolic syndrome. The main purpose of the establishment of the concept of metabolic syndrome and its treatment is to reduce the prevalence of type 2 diabetes mellitus and cardiovascular disease. The main purpose of the government policy of this health checkup and health guidance in Japan is to reduce medical expenses for lifestyle-related and cardiovascular diseases. I hope that further analysis of data accumulated during this health checkup and health guidance reveals these outcomes in the future.

\section{Conflicts of Interest}

None.

\section{Reference}

1) Grundy SM, Cleeman JI, Daniels SR, Daniel SR, Donato KA, Eckel RH, Franklin BA, Gordon DJ, Krauss RM, Savage PJ, Smith SC Jr, Spertus JA, Costa F; American Heart Association; National Heart Lung and Blood Institute: Diagnosis and management of the metabolic syndrome: an American Heart Association/National Heart, Lung and Blood Institute Scientific Statement. Circulation, 2005; 112: 2735-2752

2) Alberti KG, Zimmet PZ, Shaw P: IDF Epidemiology Consensus Group. The metabolic syndrome: a new worldwide definition. Lancet, 2005; 369: 1059-1062

3) Matsuzawa Y, Funahashi T, Nakamura Y: The concept of metabolic syndrome: Contribution of visceral fat accumulation and its molecular mechanism. J Atheroscler Thromb, 2011; 18: 629-639

4) Tsushita K, Hosler AS, Miura K, Ito Y, Fukuda T, Kitamura A Tatara K: Rationale and descriptive analysis of specific health guidance: the nationwide lifestyle intervention program targeting metabolic syndrome in Japan. J Atheroscler Thromb, 2018; 25: 308-322 